\title{
Single and double production of the Higgs boson at hadron and lepton colliders in minimal composite Higgs models
}

\author{
Shinya Kanemura,, , * Kunio Kaneta, ${ }^{2,+}$ Naoki Machida, ${ }^{1,+}$ \\ Shinya Odori,, , $\$$ and Tetsuo Shindou 3,9 \\ ${ }^{1}$ Department of Physics, University of Toyama, \\ 3190 Gofuku, Toyama, 930-8555, Japan \\ ${ }^{2}$ Center for Theoretical Physics of the Universe, \\ Institute for Basic Science (IBS), Daejeon 34051, Republic of Korea \\ ${ }^{3}$ Division of Liberal-Arts and Department of Applied Physics, Kogakuin University, \\ 1-24-2 Nishi-Shinjuku, Tokyo, 163-8677, Japan
}

\begin{abstract}
In the composite Higgs models, originally proposed by Georgi and Kaplan, the Higgs boson is a pseudo Nambu-Goldstone boson (pNGB) of spontaneous breaking of a global symmetry. In the minimal version of such models, global $\mathrm{SO}(5)$ symmetry is spontaneously broken to $\mathrm{SO}(4)$, and the pNGBs form an isospin doublet field, which corresponds to the Higgs doublet in the Standard Model (SM). Predicted coupling constants of the Higgs boson can in general deviate from the SM predictions, depending on the compositeness parameter. The deviation pattern is determined also by the detail of the matter sector. We comprehensively study how the model can be tested via measuring single and double production processes of the Higgs boson at LHC and future electronpositron colliders. The possibility to distinguish the matter sector among the minimal composite Higgs models is also discussed. In addition, we point out differences in the cross section of double Higgs boson production from the prediction in other new physics models.
\end{abstract}

\footnotetext{
*Electronic address: kanemu@sci.u-toyama.ac.jp

${ }^{\dagger}$ Electronic address: kaneta@ibs.re.kr

${ }^{\ddagger}$ Electronic address: machida@jodo.sci.u-toyama.ac.jp

${ }^{\S}$ Electronic address: odri@jodo.sci.u-toyama.ac.jp

『Electronic address: shindou@cc.kogakuin.ac.jp
} 


\section{INTRODUCTION}

By measurements of the Higgs boson property at the LHC Run-I [1, 2], it has been found that the Standard Model (SM), including the sector of electroweak symmetry breaking, is surely a good description of the physics at the electroweak scale. However the essence of the Higgs boson has not been understood yet, and it is worth asking whether the Higgs boson is really an elementary scalar or a composite state of more fundamental fields.

Although the idea of the Higgs boson as an elementary scalar field may be justified in the framework of supersymmetry (SUSY) at the TeV scale, predicted superpartner particles have not been discovered up to now at LHC. In this situation, motivation for a scenario of the Higgs boson as a composite state becomes stronger.

In particular, with the measured mass of the Higgs boson to be $125 \mathrm{GeV}$, it is interesting to consider the scenario originally proposed by Gerogi and Kaplan[3], where the Higgs boson is a pseudo Nambu-Goldstone boson (pNGB) of spontaneous symmetry breaking of a global symmetry above the $\mathrm{TeV}$ scale. Models based on this idea are called as composite Higgs models, which draw a lot of attention recently [4, 5].

The minimal composite Higgs model (MCHM)[4] is known as a minimal realisation of the composite Higgs models, where the approximate global symmetry $\mathrm{SO}(5) \times \mathrm{U}(1)_{X}$ is spontaneously broken into $\mathrm{SO}(4) \times \mathrm{U}(1)_{X}$ at the scale $f$. Associated with the symmetry breaking, four pNGBs appear, which are identified as the four component fields in the Higgs doublet of the SM. There are several attempts to probe the MCHMs by phenomenological study at the present and future collider experiments [7-12].

The induced Higgs potential depends on which representations of $\mathrm{SO}(5)$ the matter fermions are embedded into. The Higgs boson couplings with the matter fermions such as Yukawa couplings are also determined by the representations of the matter fermions. In the literature, different patterns of the deviations of the coupling constants from the SM predictions are shown in different MCHMs [8, 9]. Therefore, measuring the deviation pattern at collider experiments is critical to distinguish a variety of the MCHMs.

In this paper, we investigate collider phenomenology of the MCHMs. Because of the deviations of the Higgs boson couplings from the SM prediction, the production cross section and the branching ratios of the Higgs boson are different from those in the SM. We first clarify the constraint on the compositeness parameter in the MCHMs at the LHC Run-I. We 
then discuss double Higgs boson production at LHC and future electron-positron colliders, taking into account the constraint.

At the LHC Run-I, single Higgs boson production has been measured in various decay modes, and no significant deviation from the SM has been detected yet. In the literature[13], the constraint on the compositeness parameter is obtained in the case of the universal scale factors for the Yukawa couplings. In models with non-universal scale factors, the constraint should be extracted from the signal strength of individual production and decay mode measured at the LHC Run-I[2].

With taking into account the constraint, we evaluate cross sections of double Higgs boson production at LHC with the collision energy of $14 \mathrm{TeV}$ in several MCHMs, and discuss the possibility to distinguish among different MCHMs. The search for double Higgs boson production process at LHC can access to the coupling deviation which does not contribute to the single Higgs boson production. While there have already been several phenomenological studies of the double Higgs boson production at LHC in the context of MCHMs [10], we here study the process from the viewpoint of the discrimination of the matter sector in various MCHMs.

The double Higgs boson production process at future electron-positron colliders such as the International Linear Collider (ILC) [14] and the Compact LInear Collider (CLIC) [15] can give complementary information of the new physics. At the electron-positron colliders, the double Higgs boson production cross section can be measured as a function of the collision energy. It is known that the production cross section has a specific energy dependence on new physics models[16]. We estimate production cross sections for double Higgs boson production processes in MCHMs, and show their energy dependences.

This article is organised as follows. In Sec. II, we give a brief review of the framework of the MCHM. We introduce three different MCHMs as benchmark models, and list deviation patterns in Higgs boson coupling constants from the SM predictions and decay branching ratios of the Higgs boson. In Sec. III, we show the present bound on the model parameter from the LHC Run-I data, and we discuss the double Higgs boson production process at LHC and a future electron-positron collider experiment. Summary and conclusion are shown in Sec. IV. 


\section{HIGGS BOSON COUPLINGS IN VARIOUS MCHMS}

In this section, we give a brief review on the MCHM for self consistency. In the MCHM[4, 5], four components of the SM-like Higgs boson doublet are identified with the four pNGBs associated with the spontaneous symmetry breaking of $\mathrm{SO}(5) \times \mathrm{U}(1)_{X} \rightarrow$ $\mathrm{SO}(4) \times \mathrm{U}(1)_{X}$. The low energy effective Lagrangian can be constructed by utilising a nonlinear representation of the global symmetry group. Since $\mathrm{SU}(2)_{L} \times \mathrm{U}(1)_{Y}$ in $\mathrm{SO}(4) \times \mathrm{U}(1)_{X}$ is gauged, the global symmetry is explicitly broken by the gauge couplings. The matter fermions in the SM by themselves cannot consist SO(5) multiplet. They should be embedded into $\mathrm{SO}(5)$ representations, and the components of the multiplets other than the SM fermions are decoupled from the effective theory. The Yukawa coupling with the matter fermions of the SM also explicitly breaks the global SO(5) symmetry. Due to these explicit breaking effects of the global symmetry, the Higgs potential is effectively generated via the loop contributions of weak gauge bosons and the SM fermions. The SM fermion contribution $V_{\text {eff }}^{\text {fermion }}$ strongly depends on how the SM fermion fields are embedded into $\mathrm{SO}(5)$ multiplets. The detail of the model dependence of the Higgs potential in the MCHMs are given in Ref. [8, 9]. By solving the stationary condition, the Higgs field can have non-zero vacuum expectation value $(\mathrm{VEV})$.

We summarise deviations in the Higgs boson couplings from the SM predictions in the different MCHMs. In order to describe the deviation pattern in each MCHM, it is convenient to use the scale factors $\kappa_{a}$, which are defined by $\kappa_{a} \equiv g_{a} / g_{a}^{\mathrm{SM}}$, where $g_{a}$ denote the coupling constants of the Higgs boson coupling with the weak gauge bosons $V=W$ and $Z$, matter fermions and the Higgs boson itself as $a=h V V$, $h t t, h b b$, and $h h h$. For $\kappa_{h V V}, \kappa_{h t t}$ and $\kappa_{h b b}$, we simply write $\kappa_{V}, \kappa_{t}$ and $\kappa_{b}$, respectively. For $h h V V$ couplings, we introduce the parameter $c_{h h V V}=g_{h h V V} / g_{h h V V}^{\mathrm{SM}}$. In the effective theories of the MCHMs, new dimension five operators of two Higgs bosons and two fermions such as $h h t \bar{t}$ are induced. We parameterise the coupling constant for $h h t \bar{t}$ as $g_{h h t t}=c_{h h t t} m_{t} /\left(2 v^{2}\right)$. The four point interactions such as $h h V V$ and $h h t \bar{t}$ play important roles in our analysis.

In the MCHMs, the couplings between the Higgs boson and the weak gauge bosons deviate from the SM predictions as $g_{h V V}=g_{h V V}^{\mathrm{SM}} \sqrt{1-\xi}$, which leads to $\kappa_{V}=\sqrt{1-\xi}$, no matter how the SM fermions are embedded into $\mathrm{SO}(5)$ representations. It is the universal prediction of the MCHMs. Here the compositeness parameter $\xi$ is defined as $\xi \equiv v^{2} / f^{2}$ 
where $v(\simeq 246 \mathrm{GeV})$ is the $\mathrm{VEV}$ of the electroweak symmetry breaking. The coupling constants of the contact interaction $h h V V$ are also determined universal in the MCHMs as $c_{h h V V}=1-2 \xi$. The universal reduction of $c_{h h V V}$ is a notable feature of the MCHMs. In the Higgs sector with multi-doublet structure such as that in the minimal SUSY SM, the coupling constants $c_{h h V V}$ are given by the gauge coupling constants as the same as those in the SM.

On the other hand, Yukawa coupling constants with the matter fermions as well as the self-coupling of the Higgs boson are determined by the matter sector of the MCHMs. As described above, the matter fermions of the SM are embedded into a representation of $\mathrm{SO}(5)$. We can individually embed $q_{L}=\left(u_{L}, d_{L}\right), u_{R}, d_{R}, \ell_{L}=\left(\nu_{L}, e_{L}\right)$ and $e_{R}$. Depending on the choices for charge assignment for fermions, a variety of the models can be constructed. The patterns of scale factors in various MCHMs are listed in Refs. [8, 9].

In order to demonstrate how to distinguish the MCHMs, we pick up three typical models, MCHM4, MCHM5 and MCHM14, in which all the matter fermions are embedded into the four-, five-, and fourteen-dimensional representations of $\mathrm{SO}(5)$, respectively. The relevant matter part of the effective Lagrangian in each model is given by

$$
\begin{aligned}
\mathcal{L}_{\text {MCHM4 }}^{\text {matter }}= & \sum_{r=q, \ell, u, d, e} \bar{\Psi}_{r}^{(4)} \not p\left[\Pi_{0}^{r}+\Pi_{1}^{r} \Gamma^{i} \Sigma_{i}\right] \Psi_{r}^{(4)}+\bar{\Psi}_{q}^{(4)}\left[M_{0}^{t}+M_{1}^{t} \Gamma^{i} \Sigma_{i}\right] \Psi_{t}^{(4)} \\
& +\bar{\Psi}_{q}^{(4)}\left[M_{0}^{b}+M_{1}^{b} \Gamma^{i} \Sigma_{i}\right] \Psi_{b}^{(4)}+\bar{\Psi}_{\ell}^{(4)}\left[M_{0}^{\tau}+M_{1}^{\tau} \Gamma^{i} \Sigma_{i}\right] \Psi_{\tau}^{(4)}+\text { h.c. }, \\
\mathcal{L}_{\text {MCHM } 5}^{\text {matter }}= & \sum_{r=q, \ell, u, d, e} \bar{\Psi}_{r}^{(5)}\left[\not p \Pi_{0}^{r}+\Sigma^{\dagger} \not p \Pi_{1}^{r} \Sigma\right] \Psi_{r}^{(5)}+\bar{\Psi}_{q}^{(5)}\left[M_{0}^{t}+\Sigma^{\dagger} M_{1}^{t} \Sigma\right] \Psi_{t}^{(5)} \\
& +\bar{\Psi}_{q}^{(5)}\left[M_{0}^{b}+\Sigma^{\dagger} M_{1}^{b} \Sigma\right] \Psi_{b}^{(5)}+\bar{\Psi}_{\ell}^{(5)}\left[M_{0}^{\tau}+\Sigma^{\dagger} M_{1}^{\tau} \Sigma\right] \Psi_{\tau}^{(5)}+\text { h.c. }, \\
\mathcal{L}_{\text {MCHM14 }}^{\text {matter }}= & \sum_{r=q, \ell, u, d, e}\left[\bar{\Psi}_{r}^{(14)} \not p \Pi_{0}^{r} \Psi_{r}^{(14)}+\left(\Sigma \bar{\Psi}_{r}^{(14)}\right) \not p \Pi_{1}^{r}\left(\Psi_{r}^{(14)} \Sigma^{\dagger}\right)+\left(\Sigma_{r}^{(14)} \Sigma^{\dagger}\right) \not p \Pi_{2}^{r}\left(\Sigma \Psi_{r}^{(14)} \Sigma^{\dagger}\right)\right] \\
& +\bar{\Psi}_{q}^{(14)} M_{0}^{t} \Psi_{t}^{(14)}+\left(\Sigma \bar{\Psi}_{q}^{(14)}\right) M_{1}^{t}\left(\Psi_{t}^{(14)} \Sigma^{\dagger}\right)+\left(\Sigma \bar{\Psi}_{q}^{(14)} \Sigma^{\dagger}\right) M_{2}^{t}\left(\Sigma \Psi_{t}^{(14)} \Sigma^{\dagger}\right) \\
& +\bar{\Psi}_{q}^{(14)} M_{0}^{b} \Psi_{b}^{(14)}+\left(\Sigma \bar{\Psi}_{q}^{(14)}\right) M_{1}^{b}\left(\Psi_{b}^{(14)} \Sigma^{\dagger}\right)+\left(\Sigma \bar{\Psi}_{q}^{(14)} \Sigma^{\dagger}\right) M_{2}^{b}\left(\Sigma \Psi_{b}^{(14)} \Sigma^{\dagger}\right) \\
& +\bar{\Psi}_{\ell}^{(14)} M_{0}^{\tau} \Psi_{\tau_{R}}^{(14)}+\left(\Sigma \bar{\Psi}_{\ell}^{(14)}\right) M_{1}^{\tau}\left(\Psi_{\tau}^{(14)} \Sigma^{\dagger}\right)+\left(\Sigma \bar{\Psi}_{\ell}^{(14)} \Sigma^{\dagger}\right) M_{2}^{\tau}\left(\Sigma \Psi_{\tau}^{(14)} \Sigma^{\dagger}\right)+\text { h.c. },
\end{aligned}
$$

where $\Sigma$ is given by

$$
\Sigma=\frac{\sin (h / f)}{h}\left(h^{1}, h^{2}, h^{3}, h^{4}, h \cot (h / f)\right), \quad h=\sqrt{h^{a} h^{a}},
$$


TABLE I: Deviations in coupling constants with the Higgs boson in MCHM4, MCHM5 and MCHM14. We here show the formulae for $\xi \ll 1$. The scale factors for more variety of MCHMs are given in Refs. [8, 9].

\begin{tabular}{|c|c|c|c|c|c|c|c|}
\hline Model & $\kappa_{V}$ & $c_{h h V V}$ & $\kappa_{h h h}$ & $\kappa_{t}$ & $\kappa_{b}$ & $\kappa_{\tau}$ & $c_{h h t t}$ \\
\hline MCHM4 & \multirow{3}{*}{$1-\frac{1}{2} \xi$} & \multirow{3}{*}{$1-2 \xi$} & $1-\frac{1}{2} \xi$ & $1-\frac{1}{2} \xi$ & $1-\frac{1}{2} \xi$ & $1-\frac{1}{2} \xi$ & $-\xi$ \\
\hline MCHM5 & & & \multirow{2}{*}{$1-\frac{3}{2} \xi$} & $1-\frac{3}{2} \xi$ & \multirow{2}{*}{$1-\frac{3}{2} \xi$} & \multirow{2}{*}{$1-\frac{3}{2} \xi$} & $-4 \xi$ \\
\hline MCHM14 & & & & $1-\frac{9 M_{1}^{t}+64 M_{2}^{t}}{6 M_{1}^{t}+16 M_{2}^{t}} \xi$ & & & $-\frac{4\left(3 M_{1}^{t}+23 M_{2}^{t}\right)}{3 M_{1}^{t}+8 M_{2}^{t}} \xi$ \\
\hline
\end{tabular}

with $h^{a}$ being the $\mathrm{pNGBs}^{1}, \Psi_{r}^{(R)}$ denotes the $R$-dimensional representation into which the SM matter fermion $r$ is embedded, $\Gamma_{i}$ are the gamma matrices in $\mathrm{SO}(5)$, and $\Pi$ 's and $M$ 's are the form factor. In the analysis, we focus on the third generation of the fermions. With this Lagrangian, the Higgs potential and the Yukawa couplings of the fermions can be computed in each model.

In Table I, we summarise a set of scale factors in the three models up to the linear term of $\xi$. Since the compositeness parameter $\xi$ should be much smaller than unity by the experimental constraints as we discuss later, the higher order terms are negligible. In MCHM14, some scale factors depend not only on $\xi$ but also on $M_{1}^{t} / M_{2}^{t}$. In the limit of $M_{2}^{t} \rightarrow 0$, the deviation pattern of the scale factors becomes the same as that in MCHM5. In the following analysis, we set $M_{1}^{t}=0$ for MCHM14 for simplicity.

The deviations in the Higgs boson couplings from the SM predictions affect the Higgs boson decay branching ratios. In Fig. 1, we show ratios of decay branching ratios of the Higgs boson in MCHM5 and MCHM14 to the SM prediction in each decay mode. In the figure, $\mathrm{BR}(h \rightarrow X X)$ is the decay branching ratio of $h \rightarrow X X$ mode $(X X=Z Z, W W, \gamma \gamma, \bar{\tau} \tau$ and $\bar{b} b)$ and $\mathrm{BR}_{\mathrm{SM}}(h \rightarrow X X)$ is its $\mathrm{SM}$ prediction. In Table II, the decay branching ratios in the SM and in the MCHMs with typical value of $\xi$ are shown. In MCHM4, all the decay widths are shifted by the same factor $\kappa_{V}^{2}=\kappa_{f}^{2}=1-\xi$. The decay branching ratios are then the same as those in the SM. In numerical evaluation, we take into account the NLO QCD corrections[17] and the NLO electroweak corrections[18] to the branching ratios.

\footnotetext{
${ }^{1}$ We take $h^{3}$ as the physical Higgs boson[4, 5].
} 

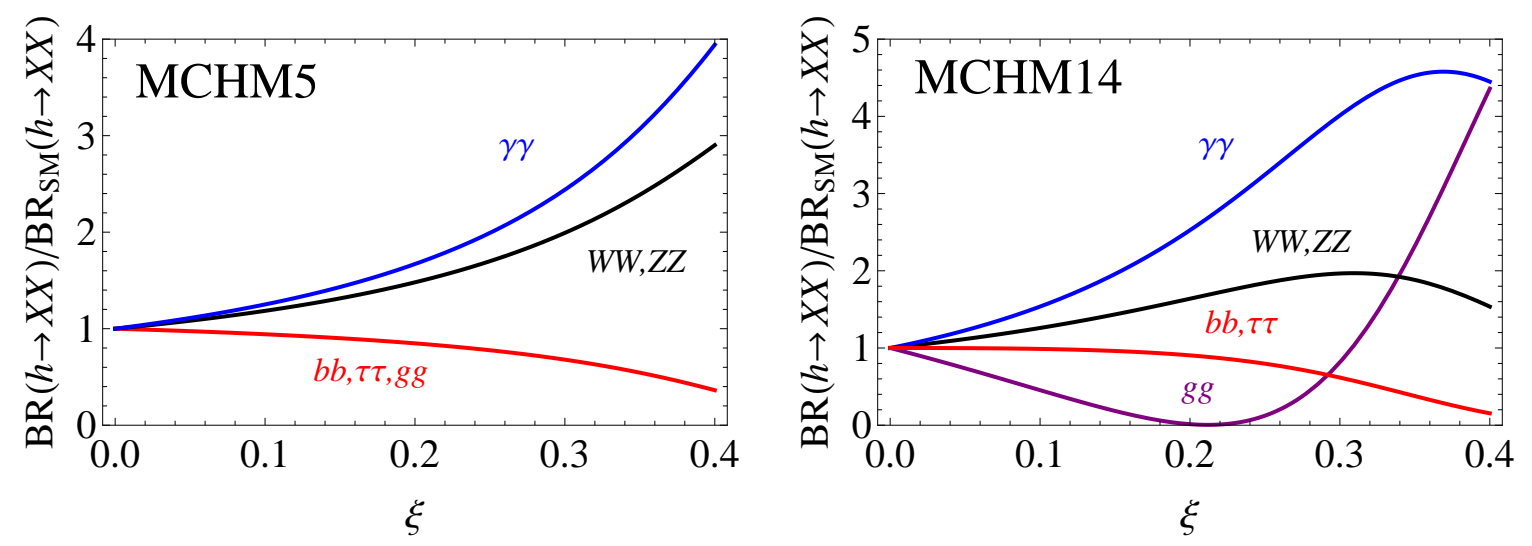

FIG. 1: Ratios of the Higgs boson decay branching ratios in MCHM5, and MCHM14 to those in the SM as a function of the compositeness parameter $\xi$.

TABLE II: The decay branching ratios of the Higgs boson in the SM, MCHM4, MCHM5, and MCHM14. For MCHM5 and MCHM14, the compositeness parameter $\xi$ is taken as $\xi=0.1$ and 0.2. The NLO QCD corrections [17] and the NLO electroweak corrections [18] are taken into account.

\begin{tabular}{|c|c|c|c|c|c|c|}
\hline Branching Ratio & $h \rightarrow b b$ & $h \rightarrow W W$ & $h \rightarrow g g$ & $h \rightarrow \tau \tau$ & $h \rightarrow Z Z$ & $h \rightarrow \gamma \gamma$ \\
\hline SM and MCHM4 & 0.555 & 0.231 & 0.0894 & 0.0674 & 0.0282 & 0.00244 \\
\hline \hline MCHM5 $(\xi=0.1)$ & 0.517 & 0.274 & 0.0843 & 0.0629 & 0.0334 & 0.00305 \\
MCHM5 $(\xi=0.2)$ & 0.457 & 0.343 & 0.0759 & 0.0556 & 0.0417 & 0.00407 \\
\hline MCHM14 $(\xi=0.1)$ & 0.548 & 0.292 & 0.0406 & 0.0668 & 0.0355 & 0.00374 \\
MCHM14 $(\xi=0.2)$ & 0.502 & 0.379 & 0.00121 & 0.0615 & 0.0461 & 0.00615 \\
\hline
\end{tabular}

\section{PHENOMENOLOGY OF THE MCHMS AT COLLIDER EXPERIMENTS}

\section{A. Current constraint on the compositeness parameter from the LHC Run-I data}

It is known that the value of the compositeness parameter $\xi$ is constrained as $\xi<0.25$ from the electroweak precision data [5, 12]. We here consider the constraint from the LHC Run-I data, which gives a significant impact. In order to take into account the constraint from the data without extra assumption, we compare the model prediction with the measurement by 
TABLE III: The signal strength in various modes measured at ATLAS and CMS. The values are taken from the results for the 10-parameter fit in Ref. [2].

\begin{tabular}{c|c|c|c}
\hline & ATLAS+CMS & ATLAS & CMS \\
\hline$\mu_{F}^{\gamma \gamma}$ & $1.19_{-0.25}^{+0.28}$ & $1.31_{-0.34}^{+0.37}$ & $1.01_{-0.31}^{+0.34}$ \\
$\mu_{F}^{Z Z}$ & $1.44_{-0.34}^{+0.38}$ & $1.73_{-0.45}^{+0.51}$ & $0.97_{-0.42}^{+0.54}$ \\
$\mu_{F}^{W W}$ & $1.00_{-0.20}^{+0.23}$ & $1.10_{-0.26}^{+0.29}$ & $0.85_{-0.25}^{+0.28}$ \\
$\mu_{F}^{\bar{\tau} \tau}$ & $1.10_{-0.58}^{+0.61}$ & $1.72_{-1.13}^{+1.24}$ & $0.91_{-0.64}^{+0.69}$ \\
$\mu_{F}^{\bar{b} b}$ & $1.09_{-0.89}^{+0.93}$ & $1.51_{-1.08}^{+1.15}$ & $0.10_{-1.86}^{+1.83}$ \\
$\mu_{V}^{\gamma \gamma}$ & $1.05_{-0.41}^{+0.44}$ & $0.69_{-0.58}^{+0.64}$ & $1.37_{-0.56}^{+0.62}$ \\
$\mu_{V}^{Z Z}$ & $0.48_{-0.91}^{+1.37}$ & $0.26_{-0.91}^{+1.60}$ & $1.44_{-2.30}^{+2.32}$ \\
$\mu_{V}^{W W}$ & $1.38_{-0.37}^{+0.41}$ & $1.56_{-0.46}^{+0.52}$ & $1.08_{-0.58}^{+0.65}$ \\
$\mu_{V}^{\bar{\tau} \tau}$ & $1.12_{-0.35}^{+0.37}$ & $1.29_{-0.53}^{+0.58}$ & $0.87_{-0.45}^{+0.49}$ \\
$\mu_{V}^{\bar{b} b}$ & $0.65_{-0.29}^{+0.30}$ & $0.50_{-0.37}^{+0.39}$ & $0.85_{-0.44}^{+0.47}$ \\
\hline
\end{tabular}

using the signal strength defined as

$$
\mu_{I}^{X X}=\frac{\sigma_{I} \cdot \mathrm{BR}(h \rightarrow X X)}{\left(\sigma_{I}\right)_{\mathrm{SM}} \cdot \mathrm{BR}_{\mathrm{SM}}(h \rightarrow X X)},
$$

where $\sigma_{I}$ is the production cross section in several processes as $I=\operatorname{ggF}$ (gluon fusion), $\mathrm{VBF}$ (vector boson fusion), $W h, Z h$ and $t t h$ (associate productions with $W, Z$, and $\bar{t} t$ respectively), and $\left(\sigma_{I}\right)_{\mathrm{SM}}$ is its SM prediction. The measured values of the signal strength in various modes with error are listed in Table III, which are taken from Ref. [2]. In this table, $\mu_{V}^{X X}$ and $\mu_{F}^{X X}$ are the abbreviations for $\mu_{\mathrm{VBF}+V h}^{X X}$ and $\mu_{\mathrm{ggF}+t h}^{X X}$, respectively. In the MCHMs, the prediction on the signal strength $\mu_{V}^{X X}$ and $\mu_{F}^{X X}$ are given by

$$
\mu_{V}^{X X}=\kappa_{V}^{2} \frac{\mathrm{BR}(h \rightarrow X X)}{\mathrm{BR}_{\mathrm{SM}}(h \rightarrow X X)}, \quad \mu_{F}^{X X}=\kappa_{t}^{2} \frac{\mathrm{BR}(h \rightarrow X X)}{\mathrm{BR}_{\mathrm{SM}}(h \rightarrow X X)} .
$$

In Fig. 2, we show the cross section of the single Higgs boson production $p p \rightarrow h X$ via gluon fusion and vector boson fusion at the LHC with $8 \mathrm{TeV}$ and $14 \mathrm{TeV}$. In the calculation for gluon fusion, we use the parton distribution function (PDF) of MSTW 2008lo[19] and the NNLO $k$-factor as $k=2.38$ for $8 \mathrm{TeV}$ and $k=2.27$ for $14 \mathrm{TeV}[20]$. The production cross section for vector boson fusion with NNLO QCD correction and the NLO electroweak correction is predicted in the $\mathrm{SM}$ as $\left(\sigma_{\mathrm{VBF}}\right)_{\mathrm{SM}}=1.58 \mathrm{pb}$ for the $8 \mathrm{TeV}$ case and $\left(\sigma_{\mathrm{VBF}}\right)_{\mathrm{SM}}=$ $4.23 \mathrm{pb}$ for the $14 \mathrm{TeV}$ case[21]. The gluon fusion process is determined by $\kappa_{t}$ so that the 


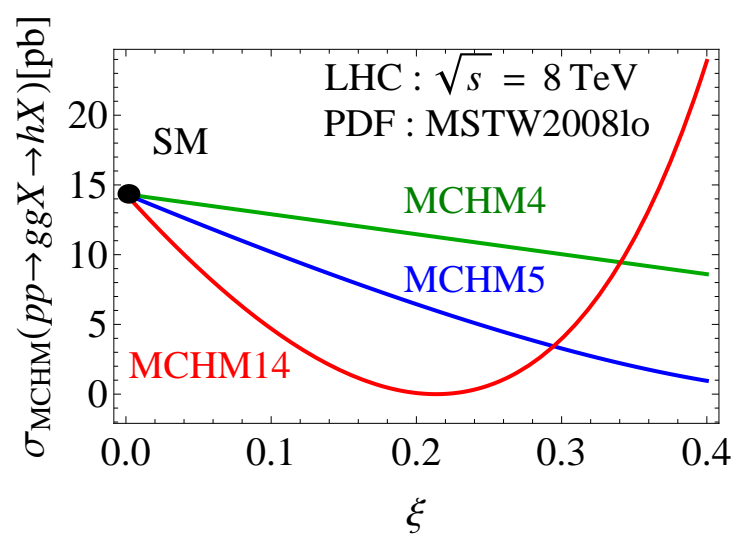

(a)

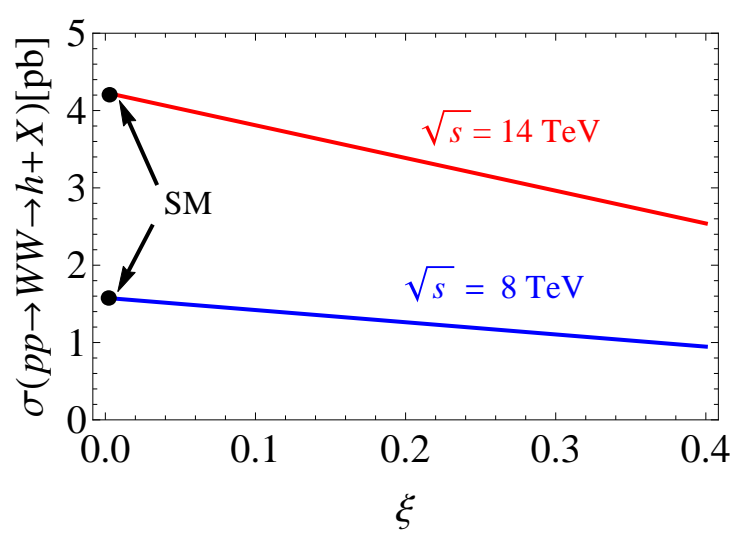

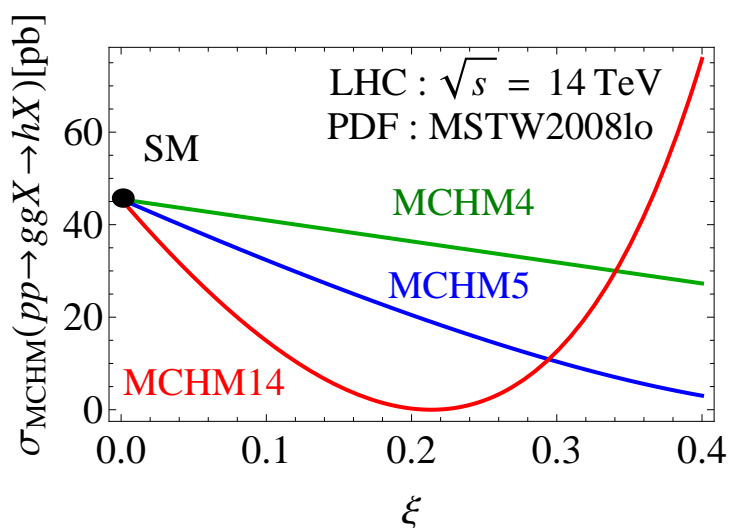

(b)

(c)

FIG. 2: (a) and (b): The production cross section of the single Higgs boson production via gluon fusion $p p \rightarrow g g X \rightarrow h X$ at LHC with (a) $8 \mathrm{TeV}$ and (b) $14 \mathrm{TeV}$ in MCHM4(Green), MCHM5(Blue) and MCHM14(Red) as a function of $\xi$. We use the $k$-factor as $k=2.38$ for the $8 \mathrm{TeV}$ case and $k=2.27$ for the $14 \mathrm{TeV}$ case. (c): the production cross section of the single Higgs boson via $W$-fusion $p p \rightarrow W^{+} W^{-} X \rightarrow h X$ at LHC with $8 \mathrm{TeV}$ (blue) and $14 \mathrm{TeV}$ (red). The cross section via $W$-fusion is universal for the MCHMs.

cross section depends on the models. On the other hand the vector boson fusion process is determined by the value of $\kappa_{V}$. The cross section for the vector boson fusion is independent of the matter sector of the MCHMs.

In Figs. 3 and 4, values of the signal strength predicted in MCHM4, MCHM5 and MCHM14 are shown. Comparing the predictions for the signal strength with the combined data of ATLAS and CMS[2], we find significant constraints on the $\xi$ for each model 
TABLE IV: The $2 \sigma$ constraint on the value of $\xi$ obtained from the ATLAS and CMS combined data at the LHC Run-I experiments in MCHM4, MCHM5, and MCHM14.

\begin{tabular}{|c|c|c|c|c|c|c|}
\hline Model & $\mu_{F}^{\gamma \gamma}$ & $\mu_{F}^{Z Z}$ & $\mu_{F}^{W W}$ & $\mu_{V}^{\gamma \gamma}$ & $\mu_{V}^{W W}$ & $\mu_{V}^{\tau \tau}$ \\
\hline MCHM4 & $\xi<0.31$ & $\xi<0.24$ & $\xi<0.40$ & - & $\xi<0.36$ & - \\
\hline MCHM5 & $\xi<0.24$ & $\xi<0.15$ & $\xi<0.23$ & $\xi<0.34$ & - & $\xi<0.30$ \\
\hline MCHM14 & $\xi<0.07$ & $\xi<0.04$ & $\xi<0.07$ & $\xi<0.19$ & - & $\xi<0.30$ \\
\hline
\end{tabular}

from $\mu_{F}^{W W}, \mu_{F}^{Z Z}, \mu_{F}^{\gamma \gamma}, \mu_{V}^{W W}, \mu_{V}^{\gamma \gamma}$ and $\mu_{V}^{\tau \tau}$ as shown in Table IV. MCHM14 is strongly constrained, because the scale factor $\kappa_{t}$ decreases rapidly when $\xi$ becomes large. In the three MCHMs, the values of the signal strength $\mu_{F}^{X X}$ are smaller than unity, while the measured values tend to be larger. In particular, the central value of $\mu_{F}^{Z Z}$ has slightly strong tension to the MCHMs prediction, so that this mode gives the strongest constraint on the compositeness parameter $\xi$, in spite of the relatively large experimental uncertainty. In the following discussion, we consider the parameter region of $\xi<0.25$ for MCHM4 and MCHM5 and $\xi<0.1$ for MCHM14.

At the LHC Run-II, expected accuracy of each signal strength is more improved to be within $10 \%$ for main channels such as $h \rightarrow \gamma \gamma, Z Z, W W, \tau \tau, b b[22]$, so that the value of $\xi$ becomes more significantly constrained.

\section{B. Double Higgs boson production at LHC}

Measuring the double Higgs boson production process provides an insight on the self coupling of the Higgs boson[23]. At the High-Luminosity LHC (HL-LHC) experiment, the cross section of the double Higgs boson production is expected to be measured with $54 \%$ uncertainty in the SM case[24]. In new physics models, the double Higgs boson production process at LHC is also important to explore the deviation pattern in the Higgs boson couplings. Differently from the single Higgs boson production or the Higgs boson decay, contact interactions such as $c_{h h V V}$ and $c_{h h t t}$ play important roles. The double Higgs boson production at LHC is analysed in the context of the MCHMs in the literature[10]. We here show our numerical results not only on the production cross section of the double Higgs boson production process, $p p \rightarrow h h X$, but also on the signal strength for each decay mode of $h h$ in MCHM4, MCHM5 and MCHM14. 

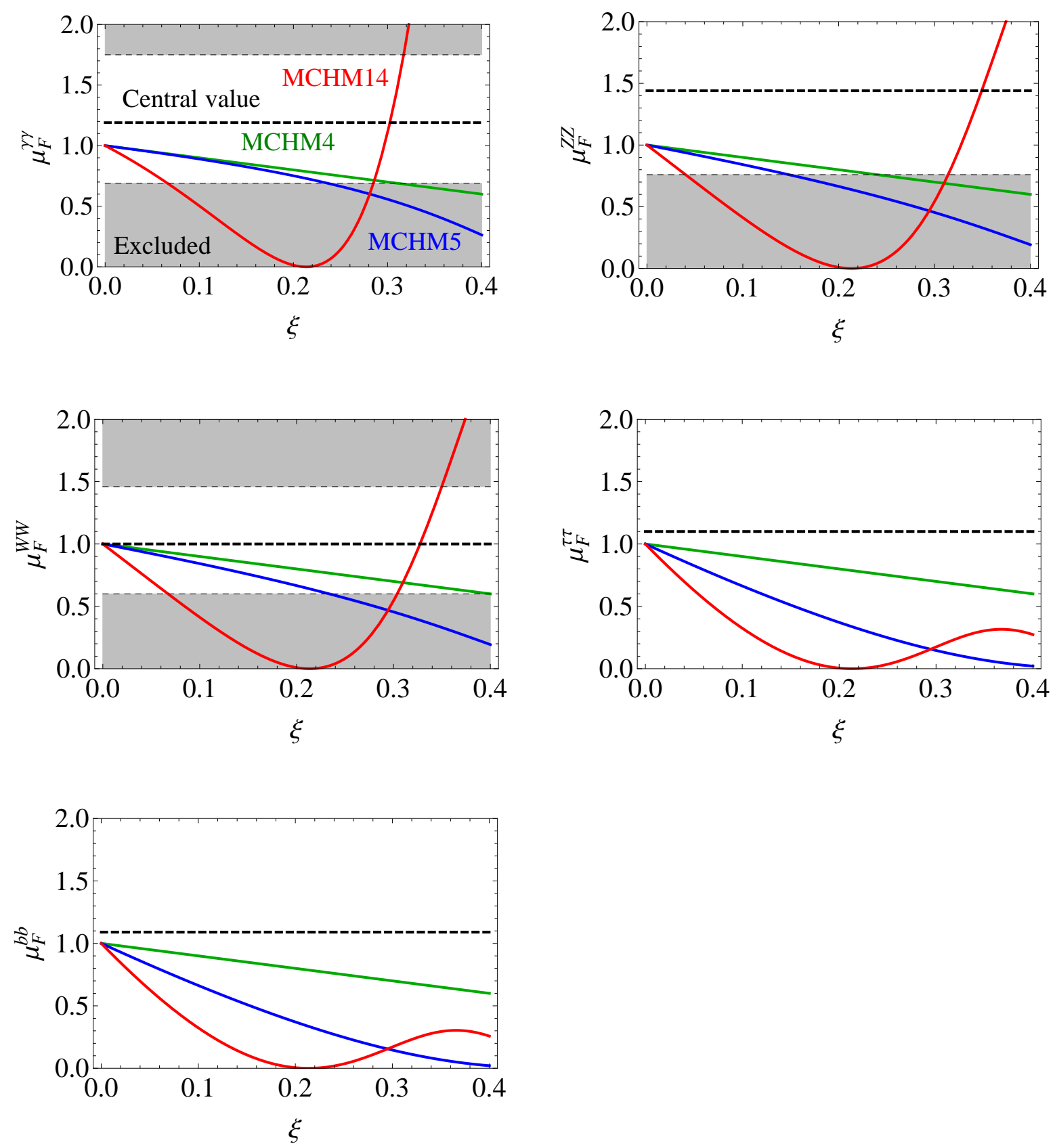

FIG. 3: The signal strength for the ggF $+t t h$ production and various decay modes $\mu_{F}^{f}$ are shown. The shaded area represents $2 \sigma$ excluded region given by the LHC Run-I data [2] in each figure.

The double Higgs boson production at LHC is dominated by the gluon fusion process, $p p \rightarrow g g X \rightarrow h h X$. The double Higgs boson production cross section via gluon fusion $p p \rightarrow h h X$ at LHC with $\sqrt{s}=14 \mathrm{TeV}$ is predicted in the $\mathrm{SM}$ as

$$
\sigma_{\mathrm{SM}}^{\mathrm{NNLO}}(p p \rightarrow g g X \rightarrow h h X)=39.1 \mathrm{fb}
$$



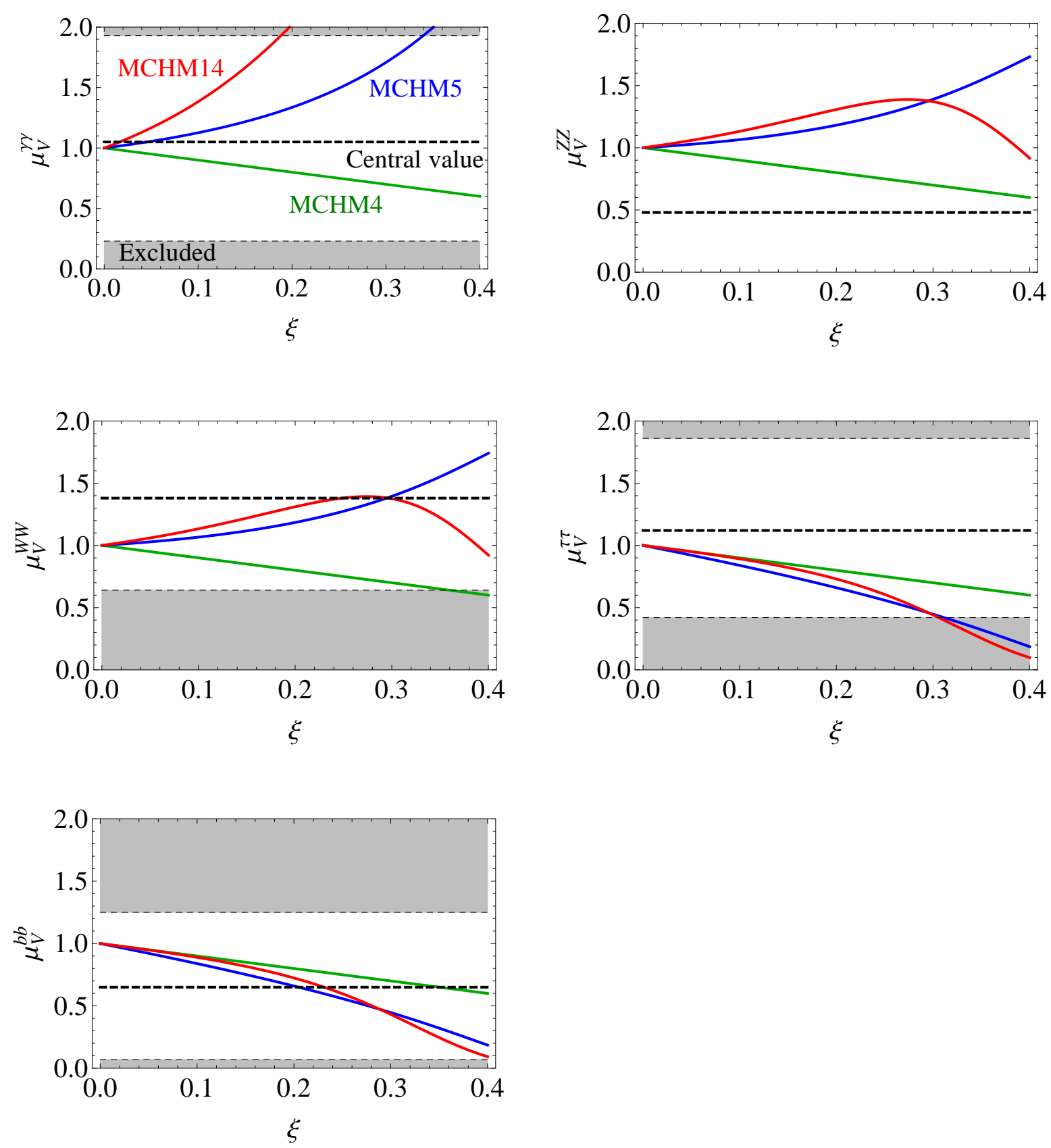

FIG. 4: The signal strength for the $\mathrm{VBF}+V h$ production and various decay modes $\mu_{V}^{f}$ are shown. The shaded area represents $2 \sigma$ excluded region given by the LHC Run-I data 2] in each figure.

where we use the PDF of MSTW 2008lo[19] and the $k$-factor at the NNLO $k=2.4[20]$. The relevant diagrams in the MCHMs are shown in Fig. 5. Deviations in the top Yukawa coupling constant and the triple Higgs boson coupling constant affect the prediction of the cross section through Fig. 5.(a), (b) and (c). In addition to these contributions, due to the 


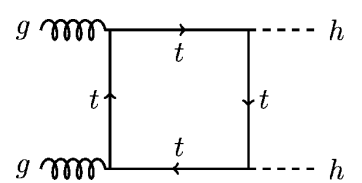

(a)

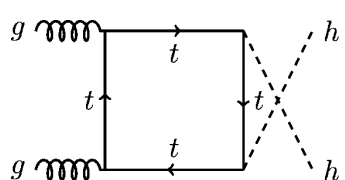

(b)

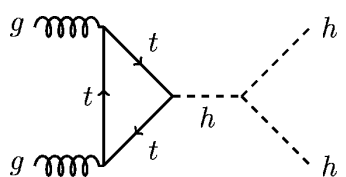

(c)

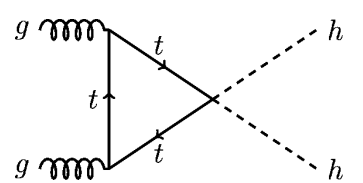

(d)

FIG. 5: Feynman diagrams for the subprocess of double Higgs boson production via gluon fusion $g g \rightarrow h h$.

existence of the dimension five interaction $h h t \bar{t}$, there is a new diagram shown in Fig. 5-(d). Therefore, the cross section of this process depends on the parameters $\kappa_{t}, \kappa_{h h h}$, and $c_{h h t t}$. This process is measured by using the decay process of $h h \rightarrow \bar{b} b \gamma \gamma$, which is expected to be the cleanest mode in the double Higgs boson production[23].

In Fig. 6, the production cross section of $p p \rightarrow g g X \rightarrow h h X$ at the LHC with $\sqrt{s}=$ $14 \mathrm{TeV}$ and the ratio of the cross section to the SM prediction are shown as a function of the compositeness parameter $\xi$ in the three different MCHMs. We also show the signal cross section $\sigma(p p \rightarrow g g X \rightarrow h h X \rightarrow \bar{b} b \gamma \gamma X)$ and the signal strength of this process $\mu$ in each model. Current upper limit is listed in Table IV, depending on models. We consider the parameter region of $\xi<0.25$ in MCHM4 and MCHM5, and $\xi<0.1$ in MCHM14.

In MCHM4, the cross section can be $10 \%$ smaller than the SM prediction for $\xi \simeq 0.25$. In MCHM5, an enhancement is found to be $15 \%$ at $\xi \simeq 0.25$. In MCHM14, the contribution in Fig. 5f(d) enhances the production cross section in the large $\xi$ region, because the size of the scale factor $c_{h h t t}=-\frac{23}{2} \xi$ is significantly large. However, in MCHM14 the value of $\xi$ is strongly constrained as $\xi<0.1$, and no significant enhancement is obtained in the allowed region of $\xi$. The signal cross section in the $\bar{b} b \gamma \gamma$ mode can be enhanced more than $65 \%$ in MCHM5 for $\xi \simeq 0.25$. Even in MCHM14, the signal cross section can be enhanced as large as $70 \%$ in the allowed region $(\xi<0.1)$ due to the significant enhancement in $\operatorname{BR}(h \rightarrow \gamma \gamma)$. Although we do not study background by ourselves, which is beyond the scope of our analysis, such a large deviation from the SM prediction may be detected at the HL-LHC where the SM prediction of the cross section of $p p \rightarrow h h X \rightarrow \gamma \gamma \bar{b} b X$ is expected to be tested with the $67 \%$ uncertainty [24].

The Higgs boson coupling with weak gauge bosons such as $\kappa_{V}$ and $c_{h h V V}$ can be explored by the double Higgs boson production via $W$-fusion. Relevant diagrams for $W$-fusion at the 


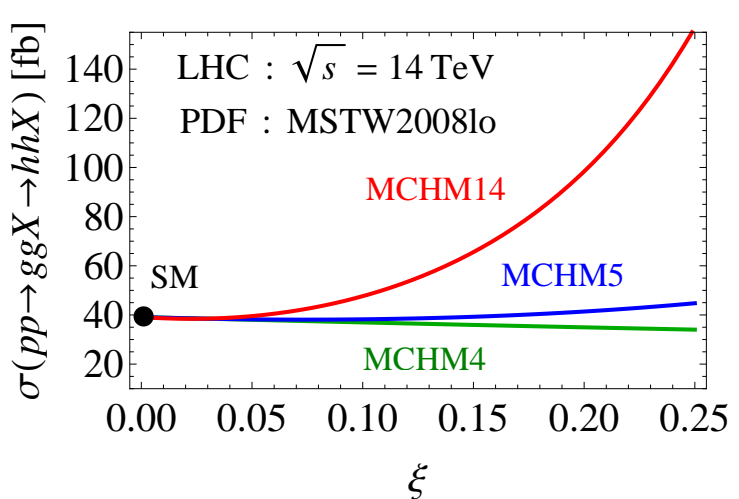

(a)

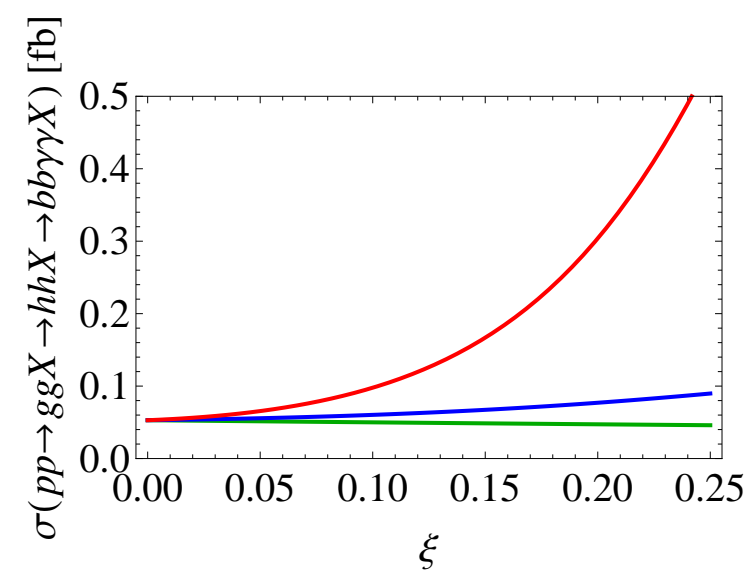

(c)

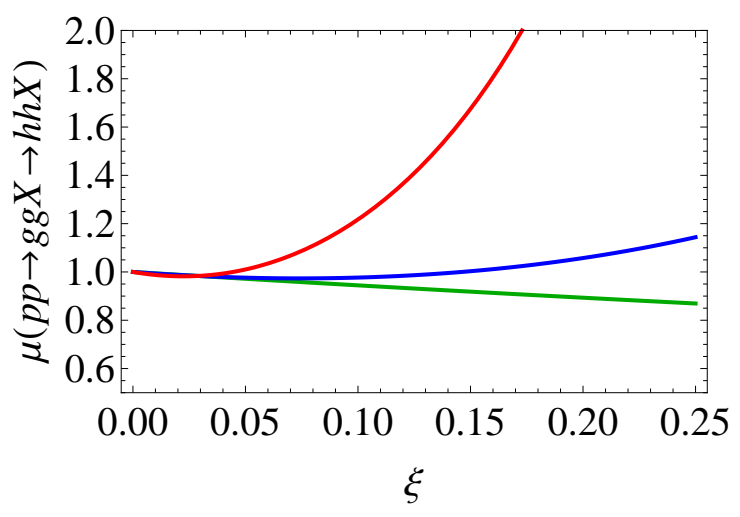

(b)

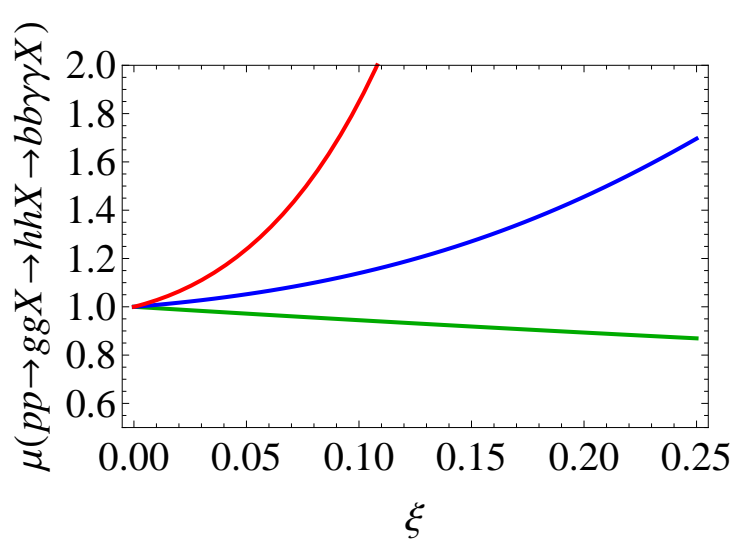

(d)

FIG. 6: (a) The production cross section of $p p \rightarrow g g X \rightarrow h h X$ in MCHM4(green), MCHM5(blue) and MCHM14(red) at LHC with the collision energy of $14 \mathrm{TeV}$. The $k$-factor of $k=2.4$ is used. (b) The ratio of the production cross section in each model to the SM value. (c) The signal cross section of $p p \rightarrow g g X \rightarrow h h X \rightarrow \bar{b} b \gamma \gamma X$ in each model. (d) The signal strength of the $p p \rightarrow g g X \rightarrow h h X \rightarrow \bar{b} b \gamma \gamma X$.

leading order is shown in Fig. 7. The elementary process for $W$-fusion is $W^{+} W^{-} \rightarrow h h$. For large centre-of-mass energy of $W^{ \pm}$as $\hat{s} \gg m_{W}^{2}$, the amplitude for the various polarisation of $W^{ \pm}$is given by

$$
\mathcal{A} \simeq A_{t} \frac{\hat{s}}{\hat{t}-m_{W}^{2}}+A_{u} \frac{\hat{s}}{\hat{u}-m_{W}^{2}}+A_{0}+A_{s} \frac{\hat{s}}{m_{W}^{2}},
$$

where $A_{s}, A_{t}, A_{u}$ and $A_{0}$ are numerical factors given in Table $\mathrm{V}$. In the SM, the cancellation 


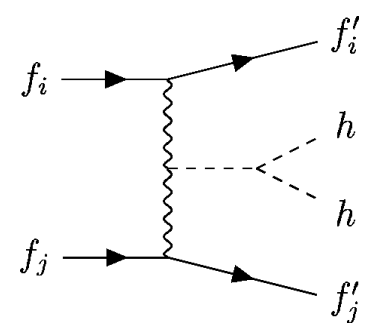

(a)

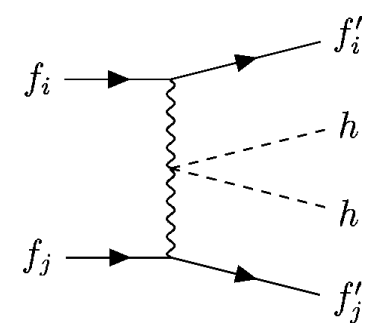

(b)

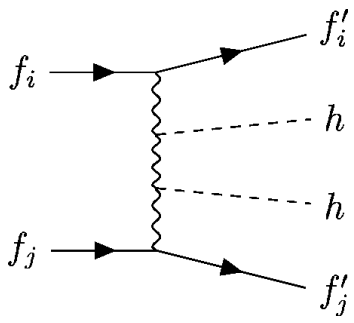

(c)

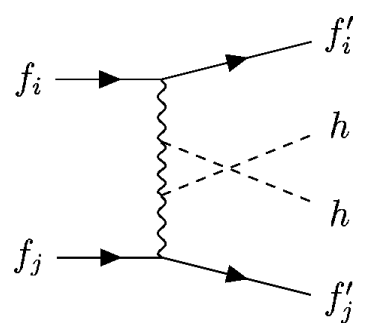

(d)

FIG. 7: Relevant diagrams for the double Higgs boson production process via $W$-fusion. For the external fermions, taking $f_{i}^{(\prime)}, f_{j}^{(\prime)}=q$ or $\bar{q}$ is for the process at LHC and taking $f_{i}=e^{-}, f_{j}=e^{+}$, $f_{i}^{\prime}=\nu$ and $f_{j}^{\prime}=\bar{\nu}$ is for an electron-positron collider.

TABLE V: The numerical factors in the scattering amplitude given in Eq. (6).

\begin{tabular}{|c|c|c|c|c|}
\hline Polarisation of $\left(W^{+}, W^{-}\right)$ & $A_{s}$ & $A_{t}$ & $A_{u}$ & $A_{0}$ \\
\hline$(0,0)$ & $\frac{g^{2}}{4}\left(c_{h h V V}-\kappa_{V}^{2}\right)$ & $\frac{g^{2} \kappa_{V}^{2}}{2}$ & $\frac{g^{2} \kappa_{V}^{2}}{2}$ & $\frac{g^{2}}{2}\left(2 \kappa_{V}^{2}-c_{h h V V}\right)+\frac{g^{2} m_{h}^{2}}{4 m_{W}^{2}}\left(3 \kappa_{V} \kappa_{h h h}-2 \kappa_{V}^{2}\right)$ \\
\hline$( \pm, \pm)$ & 0 & 0 & 0 & $\frac{g^{2}}{2}\left(c_{h h V V}-\kappa_{V}^{2}\right)$ \\
\hline$( \pm, \mp)$ & 0 & 0 & 0 & $-\frac{g^{2} \kappa_{V}^{2}}{2}$ \\
\hline
\end{tabular}

among the diagrams in Fig. 7 leads to $A_{s}=0$ so that the amplitude is at most constant in the high energy limit. On the other hand, in the MCHMs, the unitarity cancellation does not occur. In such a case, perturbative unitarity is expected to be restored at a higher scale $M$, where a new heavy resonance with the mass of $M$ may contribute to the $W^{+} W^{-} \rightarrow h h$ scattering[25]. For example, perturbative unitarity is violated at a scale of $2 \mathrm{TeV}$ for $\xi=0.25$ and at a scale of $3 \mathrm{TeV}$ for $\xi=0.1^{2}$. As discussed in Ref. [10, 25], due to this unitarity non-cancellation at the scale $\sqrt{\hat{s}}<M$, the scattering cross section of $W^{+} W^{-} \rightarrow h h$ in the MCHMs are enhanced as compared to the SM prediction, even if the relevant coupling constants are all suppressed by the scale factors. Because of this enhancement, the production cross section of $p p \rightarrow W^{+} W^{-} X \rightarrow h h X$ is enhanced.

In Fig. 8, we show the production cross section of $p p \rightarrow W^{+} W^{-} X \rightarrow h h X$ in MCHM4, MCHM5 and MCHM14, and the ratio of them to the SM prediction. Hereafter, we utilise

\footnotetext{
2 At LHC, it is rare for the centre of mass energy of two initial $W$ bosons to be higher than $2 \mathrm{TeV}$, due to the PDF suppression. Therefore it is not easy to detect the violation of the perturbative unitarity directly even in the case of $\xi=0.25$.
} 

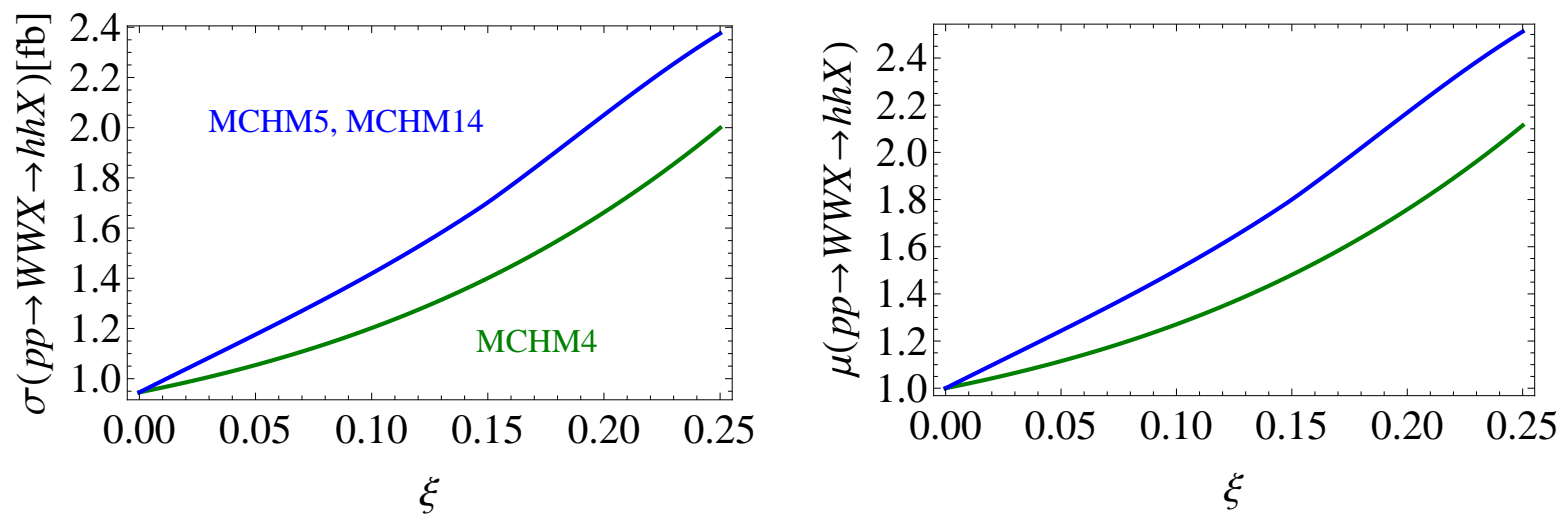

FIG. 8: Left: production cross section $p p \rightarrow W^{+} W^{-} X \rightarrow h h X$ at LHC with the collision energy of $14 \mathrm{TeV}$ as a function of $\xi$. The green curve shows the cross section in MCHM4 and the blue curve shows it in the MCHM5 and MCHM14. The $k$-factor of $k=1.0$ is used[28]. Right: The ratio of the cross section in each model to the SM value.

the CalcHep 3.6.25[26] and the CTEQ6M[27] as the PDF for the numerical computations of the cross section of the double Higgs boson production process. In the evaluation of the cross section, we use the $k$-factor as $k=1.0$ [28]. Since the relevant scale factors $\kappa_{V}, c_{h h V V}$, and $\kappa_{h h h}$ in MCHM14 are equal to those in MCHM5 as shown in Table I, the production cross section is equal to each other in these two models. By taking into account the decay modes of the Higgs boson, number of the signal event is different in MCHM5 and MCHM14.

The production cross section of $p p \rightarrow W^{+} W^{-} X \rightarrow h h X$ is as small as a few $\mathrm{fb}$ so that it is hopeless to detect the process by using the clean decay mode of $h h \rightarrow \bar{b} b \gamma \gamma$, because $\mathrm{BR}(h \rightarrow \gamma \gamma)$ is small. This process is detected in the decay modes of $h h \rightarrow \bar{b} b \bar{b} b$ and $h h \rightarrow \bar{b} b W^{+} W^{-}$, due to rather large branching ratios of $\operatorname{BR}(h \rightarrow \bar{b} b)$ and $\operatorname{BR}\left(h \rightarrow W^{+} W^{-}\right)$. In Fig. 9, the signal cross sections $\sigma\left(p p \rightarrow W^{+} W^{-} X \rightarrow h h X \rightarrow \bar{b} b \bar{b} b X\right)$ and $\sigma(p p \rightarrow$ $\left.W^{+} W^{-} X \rightarrow h h X \rightarrow \bar{b} b W^{+} W^{-} X\right)$ and their signal strength are shown. Though the signal cross sections in both modes are of order of $0.1 \mathrm{fb}$, they are larger than the SM predictions. For example, in MCHM14, the signal cross section for $\xi \simeq 0.1$ is almost twice as large as the SM prediction. Such a large enhancement might be measured at the HL-LHC. 


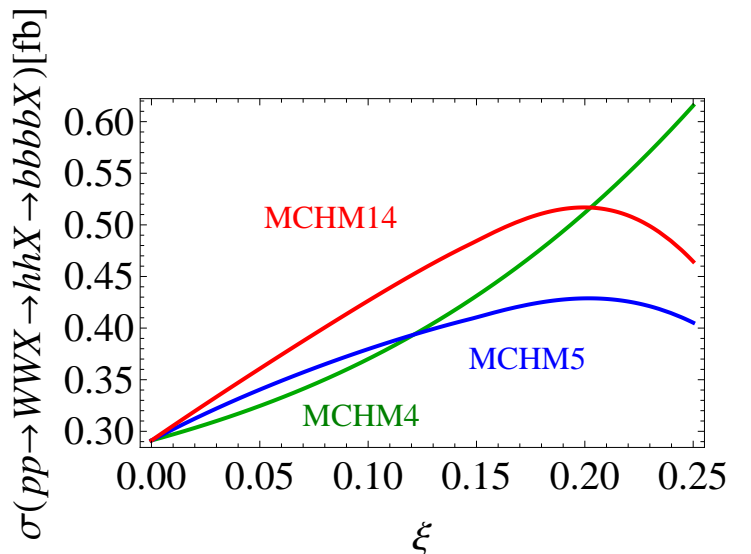

(a)

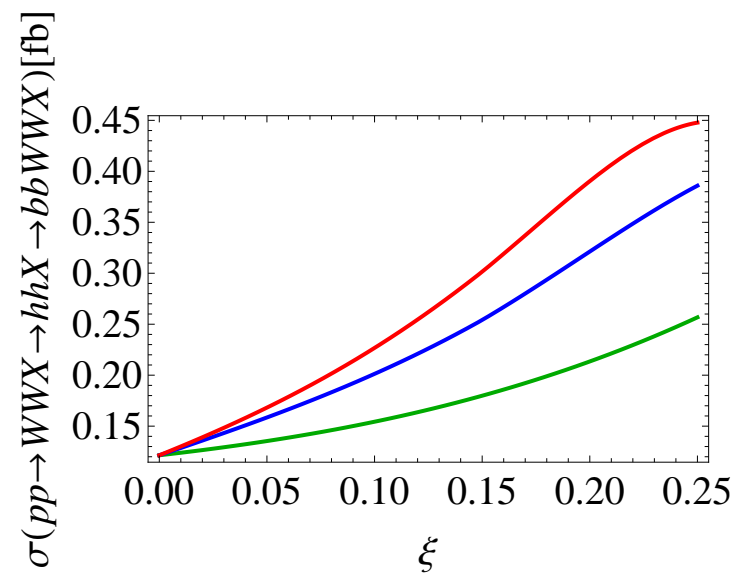

(c)

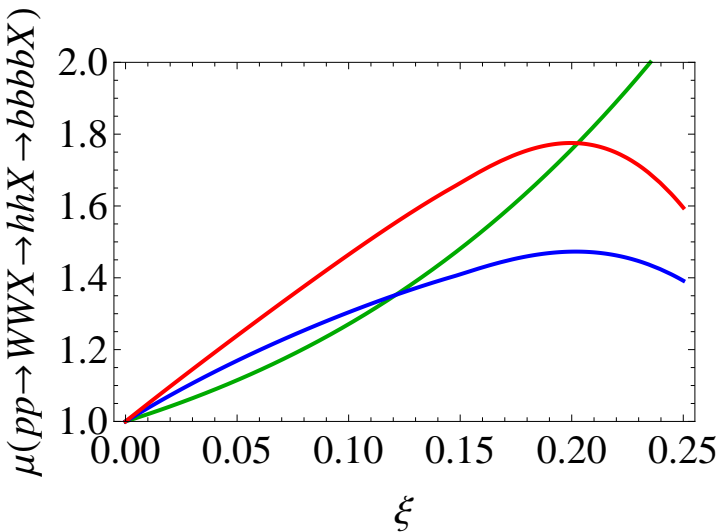

(b)

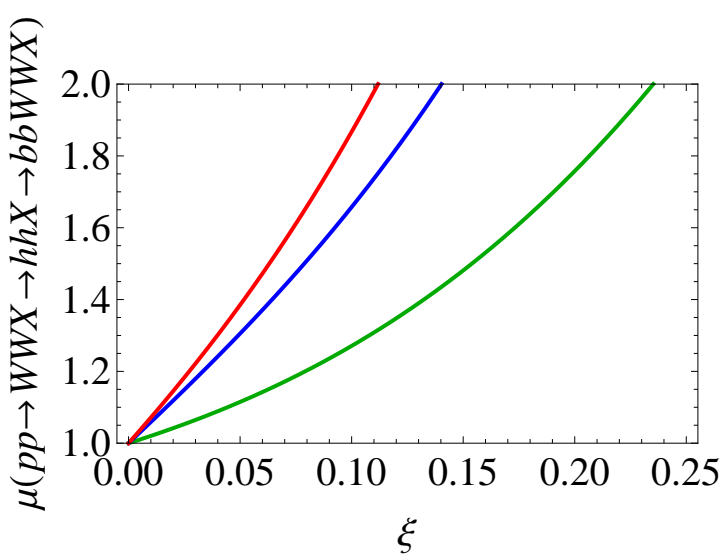

(d)

FIG. 9: (a) and (b): The signal cross section and the signal strength at LHC with the collision energy of $14 \mathrm{TeV}$ as a function of $\xi$ for the double Higgs boson production process via vector boson fusion in the decay mode of $h h \rightarrow \bar{b} b \bar{b} b$. (c) and (d): The signal cross section and the signal strength as a function of $\xi$ for the double Higgs boson production process via vector boson fusion in the decay mode of $h h \rightarrow \bar{b} b W^{+} W^{-}$. The green, blue and red curves show the predictions in MCHM4, MCHM5 and MCHM14, respectively.

\section{Double Higgs boson production at an electron-positron collider}

The scale factor $\kappa_{V}$ is very precisely measured at future electron-positron linear colliders. For example, the value of $\kappa_{V}$ is determined with accuracy of $1.0 \%$ by measuring the single Higgs boson production via $e^{+} e^{-} \rightarrow Z h$ and $e^{+} e^{-} \rightarrow h \bar{\nu} \nu$ at the ILC(500) scenario[14]. In the framework of the MCHMs, it means that the value of the compositeness parameter $\xi$ is measured at certain precision. Not only the gauge coupling of the Higgs boson but also the 
decay branching ratios of the Higgs boson is precisely measured. For example, the signal strength of the $e^{+} e^{-} \rightarrow h \bar{\nu} \nu \rightarrow \bar{b} b \bar{\nu} \nu$ is expected to be measured at $0.7 \%$ with the collision energy $\sqrt{s}=500 \mathrm{GeV}$ and the integrated luminosity of $500 \mathrm{fb}^{-1}[14]$.

The double Higgs boson production is also important to explore the Higgs sector. This process is sensitive to the triple Higgs boson coupling $h h h$ and the contact interaction $h h V V$, which cannot be determined by the single Higgs boson production processes. The most promising process for the double Higgs boson production at an electron-positron collider with $\sqrt{s}=500 \mathrm{GeV}$ is the $Z$ strahlung process as $e^{+} e^{-} \rightarrow Z^{*} \rightarrow h h Z[16$, 29]. At the ILC, two hopeful decay modes $h h \rightarrow \bar{b} b \bar{b} b$ and $h h \rightarrow \bar{b} b W^{+} W^{-}$are used to detect the double Higgs boson production process. We here discuss the production cross section. The production cross section $e^{+} e^{-} \rightarrow h h Z$ is measured with accuracy of $42.7 \%$ (23.7\%) at the ILC with $\sqrt{s}=500 \mathrm{GeV}$ and the integrated luminosity of $500 \mathrm{fb}^{-1}\left(1600 \mathrm{fb}^{-1}\right)[14]$.

In Fig. 10, the cross section of $e^{+} e^{-} \rightarrow Z h h$ and its ratio to the SM prediction is shown in MCHM4, MCHM5 and MCHM14 as a function of $\sqrt{s}$. The production cross section in MCHM14 is same as that in MCHM5. In the MCHMs the relevant coupling constants to the decay process of $Z^{*} \rightarrow h h Z$ are all suppressed by the scale factors $\kappa_{V}, c_{h h V V}$ and $\kappa_{h h h}$ so that the production cross section of $e^{+} e^{-} \rightarrow Z^{*} \rightarrow h h Z$ is suppressed, compared to the SM prediction in the all range of $\sqrt{s}$. The suppression factor is smaller than 0.5 for $\xi \gtrsim 0.2$.

The double Higgs boson production process with a missing momentum as $e^{+} e^{-} \rightarrow h h \bar{\nu} \nu$ becomes more important for higher $\sqrt{s}[16,30]$. It is expected that the production cross section is measured with the accuracy of $26.3 \%(16.7 \%)$ at the ILC with $\sqrt{s}=1 \mathrm{TeV}$ and the integrated luminosity of $1 \mathrm{ab}^{-1}\left(2.5 \mathrm{ab}^{-1}\right)[14]$. This process mainly consists of two different subprocesses; i.e., $Z$-strahlung with $Z \rightarrow \bar{\nu} \nu$ and $W$-fusion as $e^{+} e^{-} \rightarrow W^{+} W^{-} \bar{\nu} \nu \rightarrow h h \bar{\nu} \nu$. The latter provides complementary information to the process of $e^{+} e^{-} \rightarrow h h Z$. In Fig. 11, we show the production cross section of the process $e^{+} e^{-} \rightarrow h h \bar{\nu} \nu$ in MCHM4, MCHM5 and MCHM14 for fixed values of the compositeness parameter $\xi=0.1$ and 0.2 as a function of $\sqrt{s}$. As shown in these figures, the cross section of $e^{+} e^{-} \rightarrow h h \bar{\nu} \nu$ is dominated by $Z$-strahlung for $\sqrt{s} \lesssim 600 \mathrm{GeV}$ and by $W$-fusion for $\sqrt{s} \gtrsim 600 \mathrm{GeV}$.

In Fig. 12, ratios of the production cross section of $e^{+} e^{-} \rightarrow h h \bar{\nu} \nu$ to the SM prediction are shown as a function of $\sqrt{s}$. As explained before, the $Z$-strahlung process is always suppressed by the scale factors in the MCHMs, on the other hand the $W$-fusion process is 

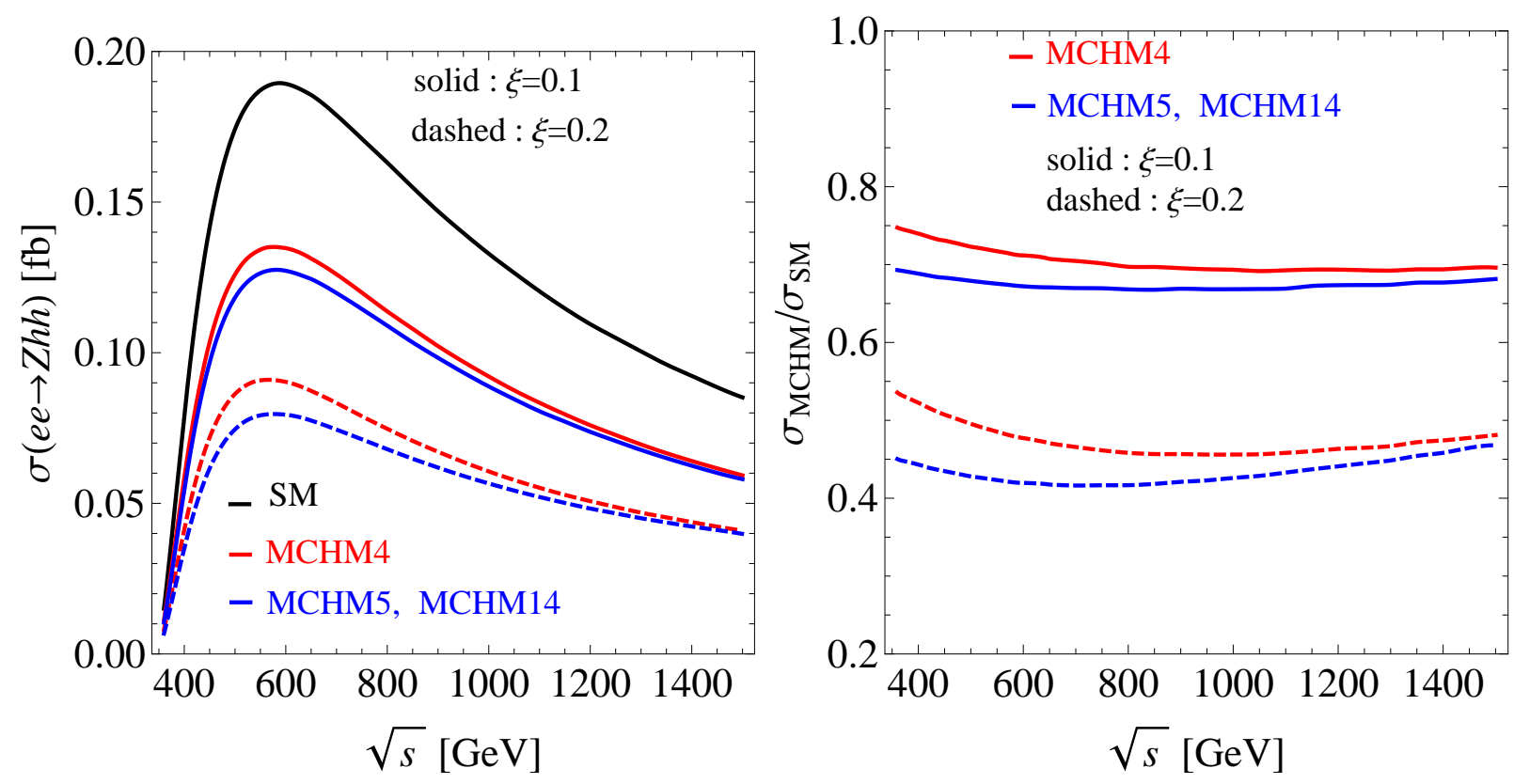

FIG. 10: Left: The cross section of $e e \rightarrow Z h h$. The black solid line shows the SM. The red (blue) solid line shows MCHM4 (MCHM5 and MCHM14). The solid (dashed) curves show the case of $\xi=0.1(\xi=0.2)$. Right: Ratios of the cross sections to the SM prediction in each model.

enhanced in the high energy region because of unitarity non-cancellation. The cross section of $e^{+} e^{-} \rightarrow h h \bar{\nu} \nu$ is then suppressed as compared to the SM prediction for $\sqrt{s} \ll 600 \mathrm{GeV}$ and it is enhanced for $\sqrt{s} \gg 600 \mathrm{GeV}$. Thanks to the expected accuracy of measurements[14, 15], such a specific behaviour might be observed by the $\sqrt{s}$ scan at the ILC and the CLIC unless the compositeness parameter $\xi$ is too small.

The $\sqrt{s}$ dependence of the double Higgs boson production cross section in the MCHMs is different from that in other new physics models. In Ref. [16], the production cross section of $e^{+} e^{-} \rightarrow h h \bar{\nu} \nu$ and $e^{+} e^{-} \rightarrow h h Z$ are discussed in the new physics models such that the triple Higgs boson couplings can significantly deviate from the SM prediction. For example, in Fig. 13, which is taken from Ref. [16], the production cross sections in the two Higgs doublet model ${ }^{3}$ are shown. In the two Higgs doublet model, due to large enhancement of the triple Higgs boson coupling by loop contributions of the extra Higgs bosons, the

$\overline{3}$ Here we focus on a scenario with the large non-decoupling loop effect [31], in which the triple Higgs boson coupling is significantly enhanced. Such a scenario can play an important role in a successful electroweak baryogenesis scenario 32 . 

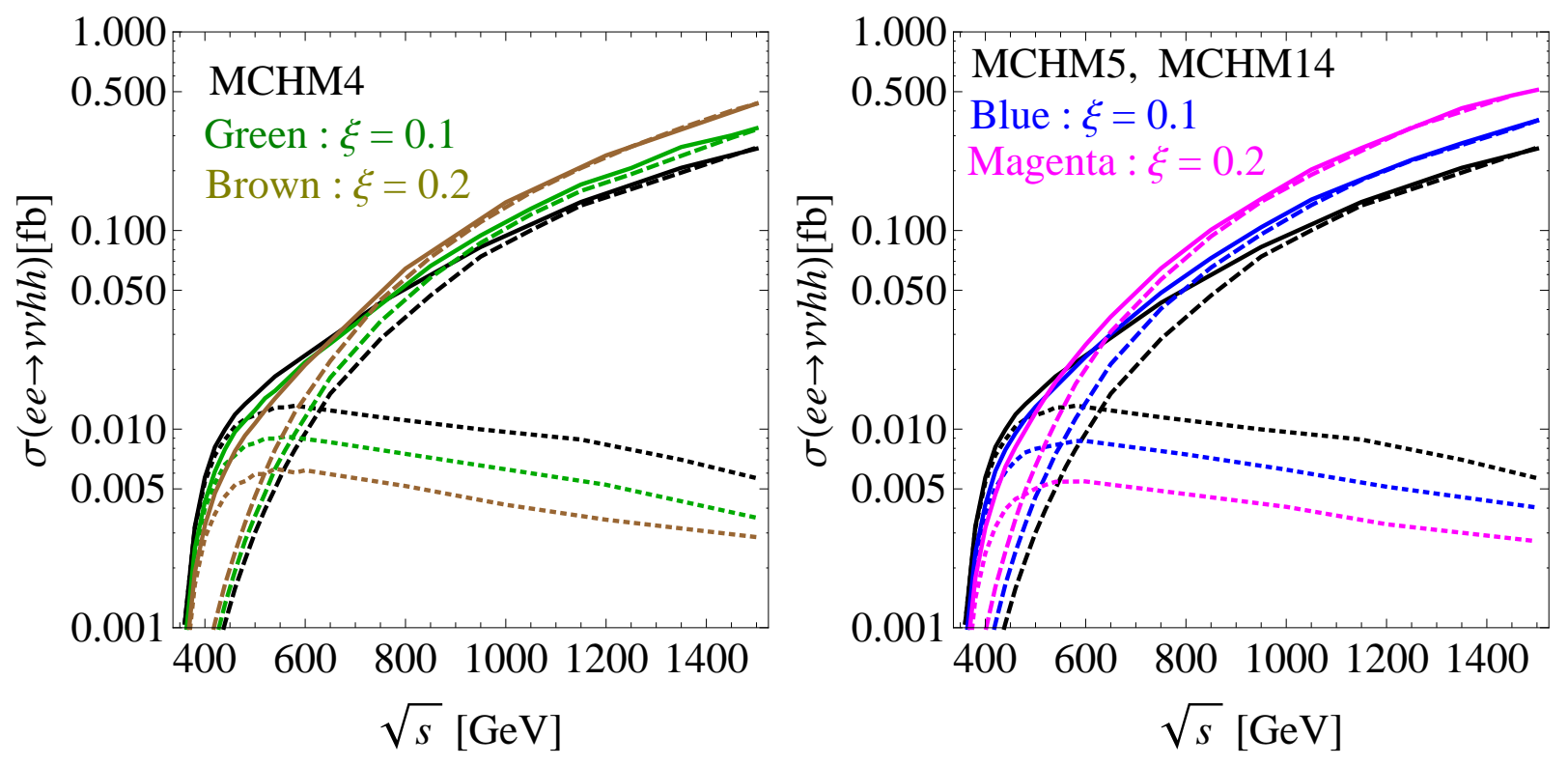

FIG. 11: Left: The cross sections for $e^{+} e^{-} \rightarrow \bar{\nu} \nu h h$ in MCHM4. The solid curve is for the total cross section. The green and the brown curves are for the case of $\xi=0.1$ and $\xi=0.2$, respectively. Right: The cross section of $e^{+} e^{-} \rightarrow \bar{\nu} \nu h h$ in MCHM5 and MCHM14. The blue and the magenta curves are for the case of $\xi=0.1$ and $\xi=0.2$, respectively. In the both figures, the dashed and dotted curves show the $W$-fusion and the $Z$-strahlung subprocesses, respectively, and the black curves show the SM prediction.

double Higgs boson production cross section via the $Z$-strahlung process can be enhanced, while the double Higgs boson production via $W$-fusion can be suppressed. In the MCHMs, on the other hand, not only the triple Higgs boson coupling but also the gauge couplings are deviated from those in the SM. All the relevant scale factors $\kappa_{h h h}, \kappa_{V}$ and $c_{h h V V}$ are suppressed, and the unitarity cancellation is incomplete. Therefore the $Z$-strahlung process is suppressed, and the $W$-fusion process is enhanced. This behaviour in the MCHMs is qualitatively different from that in the two Higgs doublet model so that these two models can be distinguished from each other.

\section{CONCLUSION}

We have investigated collider phenomenology of the MCHMs. In general, coupling constants of the Higgs boson in the MCHMs can deviate from the SM predictions. The deviation 


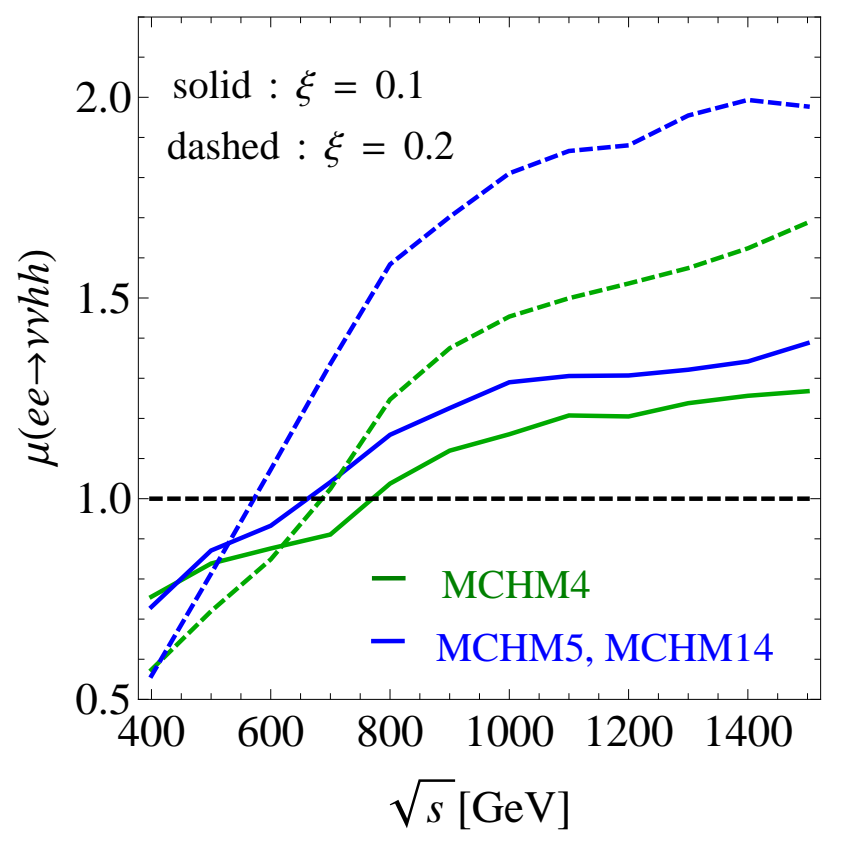

FIG. 12: The ratio of the total cross sections for $e^{+} e^{-} \rightarrow \bar{\nu} \nu h h$ in the MCHMs to that in the SM. The green (blue) curves show the prediction in MCHM4 (MCHM5 and MCHM14). The dashed and dotted curves are for the cases of $\xi=0.1$ and $\xi=0.2$, respectively.

pattern depends on the compositeness parameter as well as the detail of the matter sector.

We have comprehensively studied how the model can be tested via measuring single and double production processes of the Higgs boson at LHC and future electron-positron colliders. We have shown the model dependent constraint on the compositeness parameter $\xi$ from the LHC Run-I data. Taking into account the constraint, we have estimated the cross section of double Higgs boson production at LHC and electron-positron colliders, and discussed the possibility to distinguish the matter sector among the MCHMs. We have also shown that the $\sqrt{s}$ dependence of the double Higgs boson production cross section at electron-positron colliders differs from the prediction in other new physics models.

In summary, at the HL-LHC, a large enhancement of the signal cross section of the double Higgs boson production might be measured in some of the MCHMs. At the ILC and the CLIC, we can expect that the MCHMs are tested with higher precision unless the compositeness parameter $\xi$ is too small. Furthermore, the measurement of the double Higgs boson production process at various $\sqrt{s}$ values would be helpful to discriminate MCHMs from other new physics scenarios. 

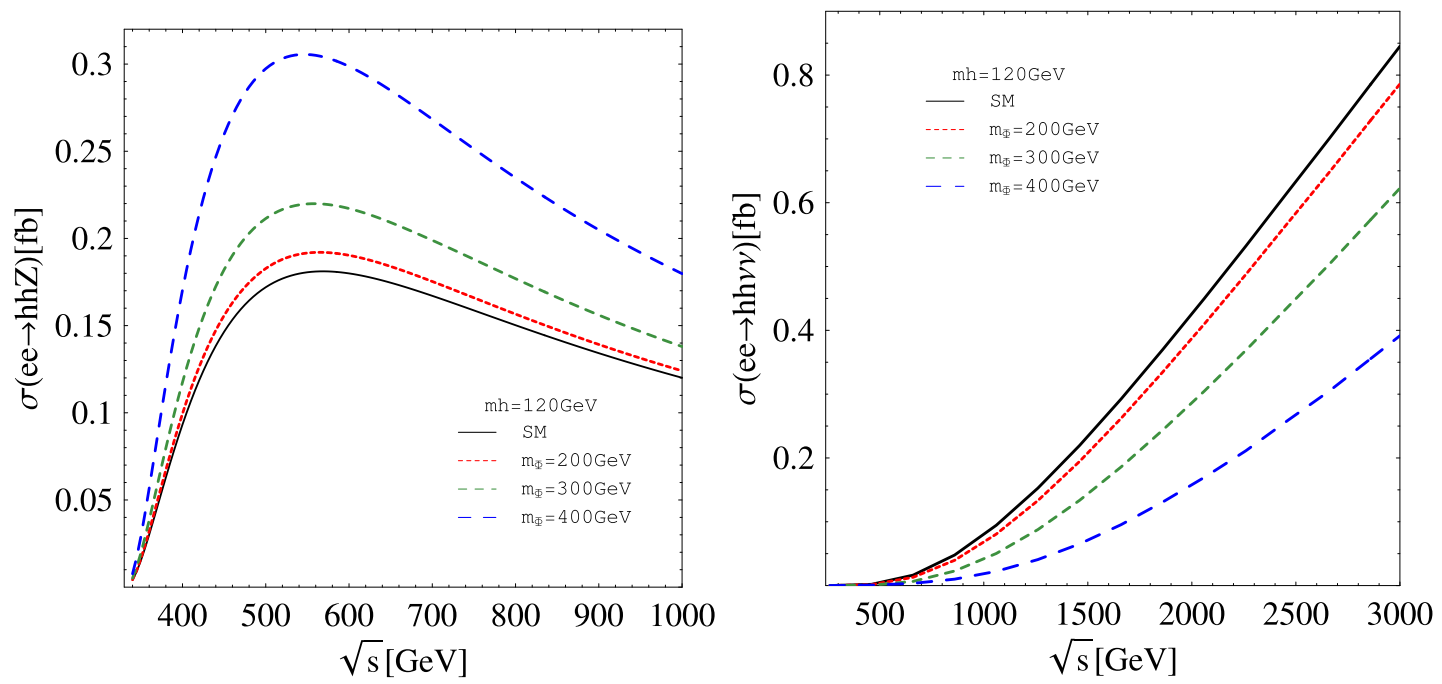

FIG. 13: Left: The cross section of $e^{+} e^{-} \rightarrow h h Z$ in the two Higgs doublet model. Right: The cross section of $e^{+} e^{-} \rightarrow h h \bar{\nu} \nu$. In the figures, the SM-like Higgs boson mass is fixed as $m_{h}=120 \mathrm{GeV}$ and the masses of extra Higgs bosons are taken to be degenerate as $m_{\Phi} \equiv m_{H}=m_{A}=m_{H^{ \pm}}$. These figures are taken from Ref. [16].

\section{Acknowledgments}

This work was supported by Grant-in-Aid for Scientific Research from the MEXT, Japan, Nos. 23104006 (S. K.) and 23104011 (T. S.), Grant H2020-MSCA-RISE-2014 No. 645722 (Non Minimal Higgs) (S. K.), and IBS under the project code IBS-R018-D1 (K. K.).

[1] G. Aad et al. [ATLAS Collaboration], Phys. Lett. B 710, 49 (2012); S. Chatrchyan et al. [CMS Collaboration], Phys. Lett. B 710, 26 (2012).

[2] The ATLAS and CMS Collaborations, ATLAS-CONF-2015-044, CMS-PAS-HIG-15-002.

[3] D. B. Kaplan and H. Georgi, Phys. Lett. B 136, 183 (1984); D. B. Kaplan, H. Georgi and S. Dimopoulos, Phys. Lett. B 136, 187 (1984); H. Georgi, D. B. Kaplan and P. Galison, Phys. Lett. B 143, 152 (1984); H. Georgi and D. B. Kaplan, Phys. Lett. B 145, 216 (1984); M. J. Dugan, H. Georgi and D. B. Kaplan, Nucl. Phys. B 254, 299 (1985).

[4] K. Agashe, R. Contino and A. Pomarol, Nucl. Phys. B 719, 165 (2005); R. Contino, L. Da Rold and A. Pomarol, Phys. Rev. D 75, 055014 (2007).

[5] R. Contino, arXiv:1005.4269 [hep-ph]; B. Bellazzini, C. Csáki and J. Serra, Eur. Phys. J. C 
74 (2014) 5, 2766.

[6] J. Mrazek, A. Pomarol, R. Rattazzi, M. Redi, J. Serra and A. Wulzer, Nucl. Phys. B 853, 1 (2011); D. Marzocca, M. Serone and J. Shu, JHEP 1208, 013 (2012); B. Gripaios, A. Pomarol, F. Riva and J. Serra, JHEP 0904, 070 (2009);

[7] G. F. Giudice, C. Grojean, A. Pomarol and R. Rattazzi, JHEP 0706, 045 (2007); A. Falkowski, Phys. Rev. D 77, 055018 (2008); J. R. Espinosa, C. Grojean and M. Muhlleitner, JHEP 1005, 065 (2010); A. Pomarol and F. Riva, JHEP 1208, 135 (2012);

A. Thamm, R. Torre and A. Wulzer, JHEP 1507, 100 (2015)

[8] M. Carena, L. Da Rold and E. Pontn, JHEP 1406 (2014) 159.

[9] S. Kanemura, K. Kaneta, N. Machida and T. Shindou, Phys. Rev. D 91 (2015) 115016.

[10] C. O. Dib, R. Rosenfeld and A. Zerwekh, JHEP 0605, 074 (2006); R. Contino, C. Grojean, M. Moretti, F. Piccinini and R. Rattazzi, JHEP 1005, 089 (2010); R. Grober and M. Muhlleitner, JHEP 1106, 020 (2011); R. Contino, M. Ghezzi, M. Moretti, G. Panico, F. Piccinini and A. Wulzer, JHEP 1208, 154 (2012); R. Grober, M. Muhlleitner and M. Spira, arXiv:1602.05851 [hep-ph].

[11] R. Contino, C. Grojean, D. Pappadopulo, R. Rattazzi and A. Thamm, JHEP 1402, 006 (2014); D. Barducci, S. De Curtis, S. Moretti and G. M. Pruna, JHEP 1402, 005 (2014).

[12] K. Agashe and R. Contino, Nucl. Phys. B 742 (2006) 59;

[13] The ATLAS collaboration [ATLAS Collaboration], ATLAS-CONF-2014-010.

[14] H. Baer et al., arXiv:1306.6352 [hep-ph]. D. M. Asner et al., arXiv:1310.0763 [hep-ph]. G. Moortgat-Pick et al., Eur. Phys. J. C 75, no. 8, 371 (2015); K. Fujii et al., arXiv:1506.05992 [hep-ex].

[15] L. Linssen, A. Miyamoto, M. Stanitzki and H. Weerts, arXiv:1202.5940 [physics.ins-det].

[16] E. Asakawa, D. Harada, S. Kanemura, Y. Okada and K. Tsumura, Phys. Rev. D 82, 115002 (2010).

[17] R. Harlander and P. Kant, JHEP 0512, 015 (2005).

[18] G. Degrassi and F. Maltoni, Nucl. Phys. B 724, 183 (2005); R. Bonciani, G. Degrassi and A. Vicini, Comput. Phys. Commun. 182, 1253 (2011).

[19] A. D. Martin, W. J. Stirling, R. S. Thorne and G. Watt, Eur. Phys. J. C 63, 189 (2009).

[20] R. V. Harlander and W. B. Kilgore, Phys. Rev. Lett. 88, 201801 (2002); D. de Florian and J. Mazzitelli, Phys. Rev. Lett. 111 (2013) 201801. 
[21] LHC Higgs Cross section Working Group Collaboration, arXiv:1307.1347 [hep-ph]; https://twiki.cern.ch/twiki/bin/view/LHCPhysics/CERNYellowReportPageAt8TeV; https://twiki.cern.ch/twiki/bin/view/LHCPhysics/CERNYellowReportPageAt1314TeV

[22] The ATLAS Collaboration, ATL-PHYS-PUB-2013-014; The CMS Collaboration, arXiv:1307.7135.

[23] E. W. N. Glover and J. J. van der Bij, Nucl. Phys. B 309, 282 (1988); T. Plehn, M. Spira and P. M. Zerwas, Nucl. Phys. B 479, 46 (1996) [Nucl. Phys. B 531, 655 (1998)]; S. Dawson, S. Dittmaier and M. Spira, Phys. Rev. D 58, 115012 (1998); A. Djouadi, W. Kilian, M. Muhlleitner and P. M. Zerwas, Eur. Phys. J. C 10, 45 (1999).

[24] CMS Collaboration [CMS Collaboration], CMS-PAS-FTR-15-002.

[25] R. Contino, D. Marzocca, D. Pappadopulo and R. Rattazzi, JHEP 1110, 081 (2011).

[26] A. Belyaev, N. D. Christensen and A. Pukhov, Comput. Phys. Commun. 184, 1729 (2013).

[27] J. Pumplin, D. R. Stump, J. Huston, H. L. Lai, P. M. Nadolsky and W. K. Tung, JHEP 0207, $012(2002)$.

[28] R. Frederix, S. Frixione, V. Hirschi, F. Maltoni, O. Mattelaer, P. Torrielli, E. Vryonidou and M. Zaro, Phys. Lett. B 732, 142 (2014).

[29] G. J. Gounaris, D. Schildknecht and F. M. Renard, Phys. Lett. B 83, 191 (1979); A. Djouadi, H. E. Haber and P. M. Zerwas, Phys. Lett. B 375, 203 (1996); V. A. Ilyin, A. E. Pukhov, Y. Kurihara, Y. Shimizu and T. Kaneko, Phys. Rev. D 54, 6717 (1996).

[30] V. D. Barger and T. Han, Mod. Phys. Lett. A 5, 667 (1990).

[31] S. Kanemura, S. Kiyoura, Y. Okada, E. Senaha and C. P. Yuan, Phys. Lett. B 558, 157 (2003); S. Kanemura, Y. Okada, E. Senaha and C.-P. Yuan, Phys. Rev. D 70, 115002 (2004).

[32] S. Kanemura, Y. Okada and E. Senaha, Phys. Lett. B 606, 361 (2005). 